\title{
Perception analysis of students and faculty of a self-directed learning module in biochemistry in a north Indian medical college
}

\author{
Poonam Agrawal ${ }^{1}$, Shinky Mehta ${ }^{2}$, Niket Verma ${ }^{3 *}$ \\ ${ }^{1}$ Professor, ${ }^{2}$ Tutor, ${ }^{3}$ Assistant Professor, ${ }^{1}$ Dept. of Biochemistry \& Member Medical Education Unit, ${ }^{2}$ Dept. of Biochemistry, ${ }^{3}$ Dept. of \\ General Medicine \& Member Medical Education Unit, ${ }^{1,2}$ Dr. Baba Saheb Ambedkar Medical College, New Delhi, ${ }^{3}$ Army College of \\ Medical Sciences, Delhi Cantt., New Delhi, India
}

\section{*Corresponding Author: Niket Verma}

Email: drniketverma@gmail.com

\begin{abstract}
Introduction: Self Directed Learning (SDL) is one of the key components of adult learning theory proposed by Malcolm Knowles. SDL is an important tool for making medical students lifelong learners. Health professionals need to be self-directed learners to keep pace with exponentially increasing medical knowledge.

Aim: This study aims to study the perception of students and faculty towards a recently conducted SDL module for first phase students in Biochemistry.

Materials and Methods: The SDL was implemented in 2 sessions. In Session 1 a case scenario of jaundice was presented to the students. Students were asked to formulate learning objectives. The students were provided resource materials and a WhatsApp group was created to motivate the students for self-directed learning and for clarifying any doubts from the students. The students were given 4 days to learn the topic on their own and regular reminders of the timeline were posted on the group. One day before Session 2 students were assessed by 5 MCQs using an online quiz. Session 2 was conducted 5 days later during a 2 hour tutorial slot as a Fishbowl Session. Afterwards, the postdiscussion assessment questionnaire was distributed to students and perception analysis questionnaire was distributed to the students and faculty.

Results: An overwhelming majority of the students [84\%] have found SDL as a more interesting and enthusiastic way of learning. Majority of students found SDL an enjoyable form of learning that gives ample opportunity to interact with the faculty. Students felt that fish bowl discussion helped them to open up and speak in peer group which certainly boosted their self-esteem and confidence and helped them develop the communication skill. $86 \%$ students found that application of clinical knowledge is easier when topic is learned in SDL module.
\end{abstract}

Keywords: Curriculum, Education, Medical, Clinical Competence, Students, Medical.

\section{Introduction}

Self Directed Learning (SDL) is one of the key components of adult learning theory proposed by Malcolm Knowles. SDL is described as "A process where learner takes his or her own initiative for learning process, determines the learning need, sets the learning goals, identify resources for learning process, learn and finally assess the learning outcome". In other words, learner in SDL programme is primarily responsible for their own learning and evaluation.

The new MCI CBME curriculum has strengthened the need of implementation of SDL in medical education by proposing 20 hours of SDL in biochemistry in $1^{\text {st }}$ year MBBS programme. The prime purpose of it is to produce medical graduates who are self-directed, independent, confident, and goal oriented so that they are better decision maker in their career to meet day to day challenges.

SDL has been advocated by many as an important tool for making medical students lifelong learners so that they are competent to identify their learning needs, allocate resources and evaluate the learning process throughout their career to better equip themselves with the latest advancements in the world of medicine. Health professionals need to be self-directed learners so as to increase their independence, self-confidence, self-discipline and make them more goal oriented to keep pace with exponentially increasing medical knowledge ${ }^{1}$. SDL has been both a fascinating and an established terminology and defining SDL and agreeing to it's worth has been a challenge. ${ }^{2,3}$

\section{Aim}

This study aims to study the perception of students and faculty towards a recently conducted SDL module for first phase students in Biochemistry.

\section{Materials and Methods}

The SDL session was conducted in the department of Biochemistry as per the latest guidelines of MCI Competency Based Medical Education curriculum. Student participation was voluntary and consent was taken.

Initially, all 125 students consented to be a part of the study but 8 students did not join the $2^{\text {nd }}$ session of the SDL programme. Therefore, a total of 117 students participated in study.

The topic chosen for SDL was "Haeme degradation and Jaundice".

The SDL was implemented in two sessions as follows:

Session 1 incorporated all 125 students. It was conducted during a onehour SDL session slot. A case scenario of jaundice was presented to the students as follows-

'A 40 year old male presented to the OPD with complaints of pain in the abdomen and yellowish discolouration of the skin and eyes for the past 7 days. The 
pain is mainly in the upper right region of the abdomen and is accompanied with loss of appetite, nausea andvomiting. He also tells the doctor that he is passing dark coloured urine and pale coloured stools for the past few days. General physical examination confirms icterus (jaundice). The attending physician wants to advise certain biochemical tests to confirm the diagnosis and the type of Jaundice'

Students were asked to formulate learning objectives. They were guided by a facilitator. Students were instructed to brainstorm in small groups of 30 students each.The objectives formulated by each group were then presented before the large group. After discussing all the objectives suggested, the following six specific learning objectives (SLO's) were finalised -

At the end of the SDL session, the $1^{\text {st }}$ phase student should be able to-

1. Define jaundice

2. Describe the biochemical pathways involved in bilirubin metabolism

3. Enumerate the types of jaundice

4. Describe the clinical presentation of the different types of jaundice

5. Enumerate and describe the biochemical tests used to diagnose jaundice

6. Discuss the basic steps in management of a patient with jaundice

At the end of session 1, the students were provided resource materials which included links to relevant pubmed articles and references from standard text books of Biochemistry.

AWhatsApp group was created which was used both to motivate the students for self-directed learning and for clarifying any doubts from the students. The students were given four days to learn the topic on their own and regular reminders of the timeline were posted on the group. One day before Session 2 students were assessed by 5 MCQs using an online quiz made on Google Forms.
Session 2 was conducted 5 days after session 1. It was conducted during the two hour tutorial slot on two consecutive days as a Fishbowl Session. In our college, the class of 125 is divided into 2 batches, A and B. Each batch attends the Biochemistry tutorials on alternate days, therefore $2^{\text {nd }}$ session was attended by 60 students on Day 1 and 57 students on Day 2. The two hour (120 min) slot was divided as follows -

5 mins: placement of students in fishbowl seating arrangement

10 mins: brief introduction ofFishbowl technique of teaching learning

35 min: discussion among the students in inner circle facilitated and guided by facilitator

35 min: discussion among the students in outer circle facilitated and guided by facilitator

20 min: discussion and summarization by facilitator

15 min: questionnaire to students followed by dispersal

Same exercise was repeated for Batch B students next day and their tutorial slot of $2 \mathrm{hrs}$ was utilized in the similar manner as for batch A.

Fishbowl method of small group discussion was incorporated and all the students were given ample opportunity to discuss the topic learned by them under supervision and guidance by the facilitator. After the discussion was over, post-discussion assessment questionnaire and the questionnaire to assess perception analysis were distributed to the students.

The perception analysis questionnaire was also distributed to the faculty also to assess their perception regarding the SDL held.

\section{Results}

Student perception: Perception analysis of students is represented in figures $1-5$.

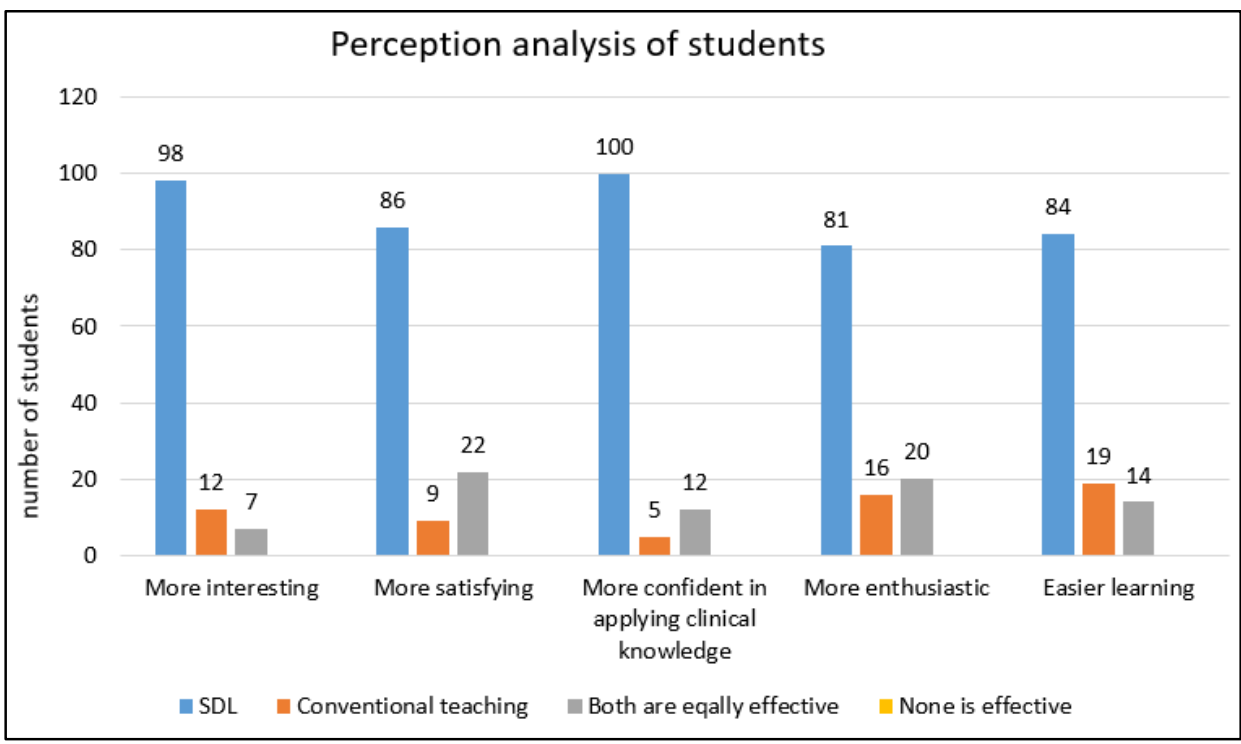

Fig. 1 


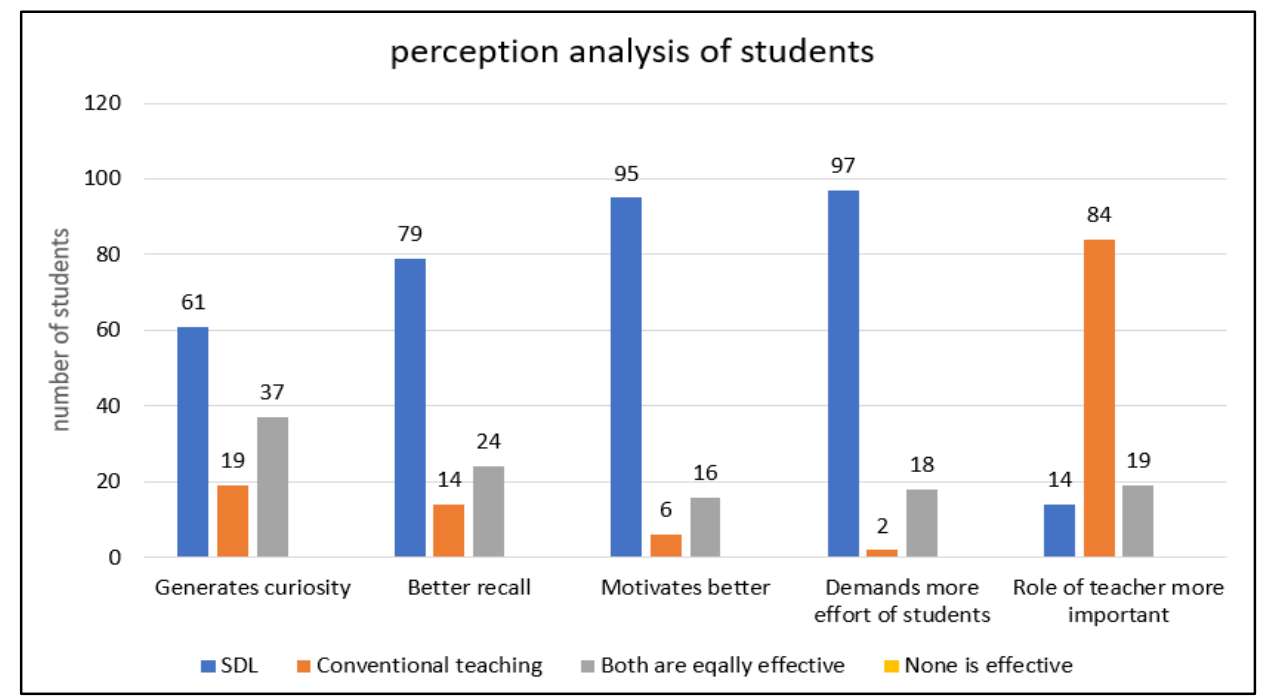

Fig. 2

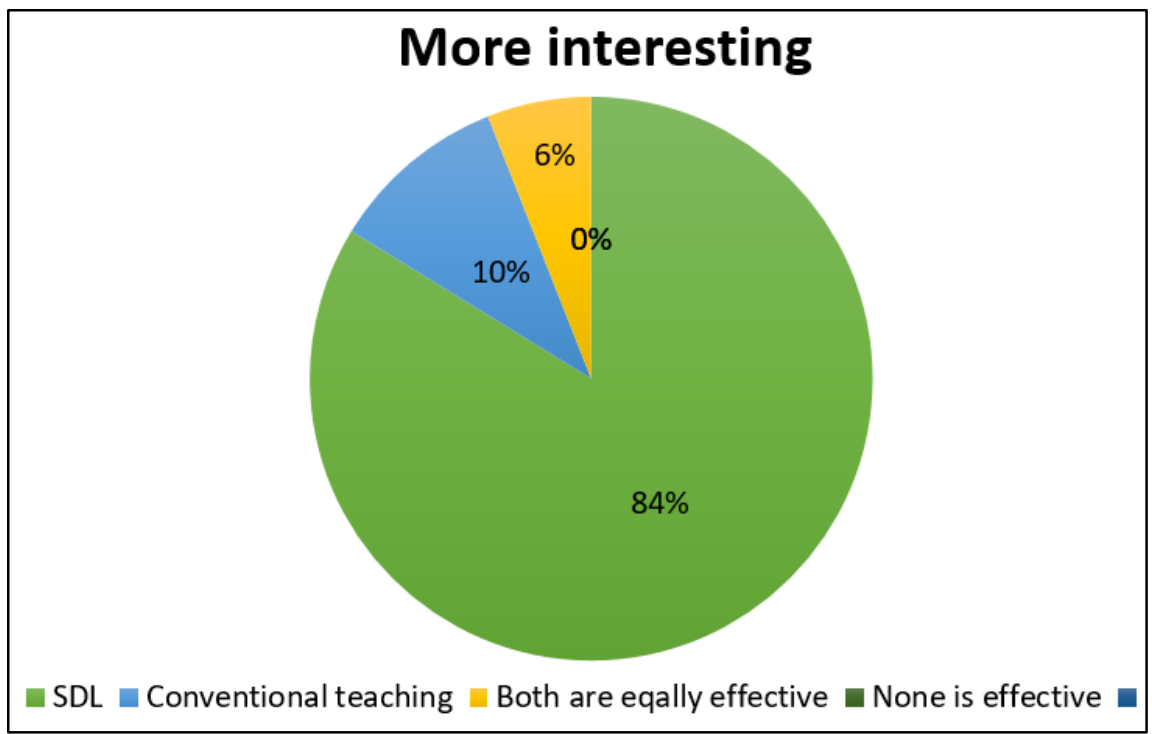

Fig. 3

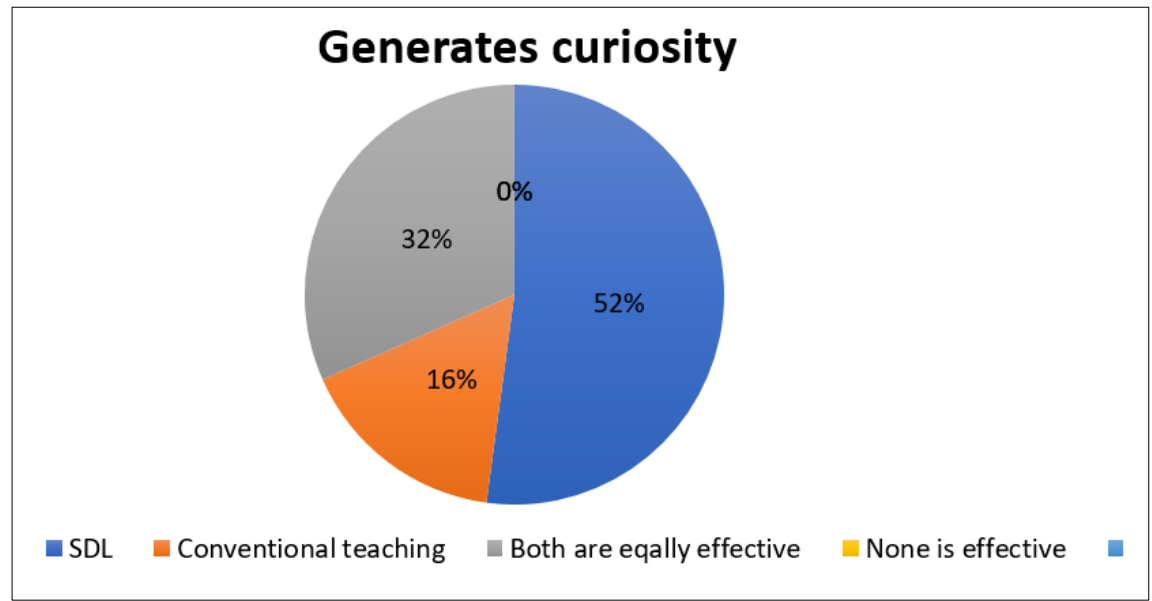

Fig. 4 


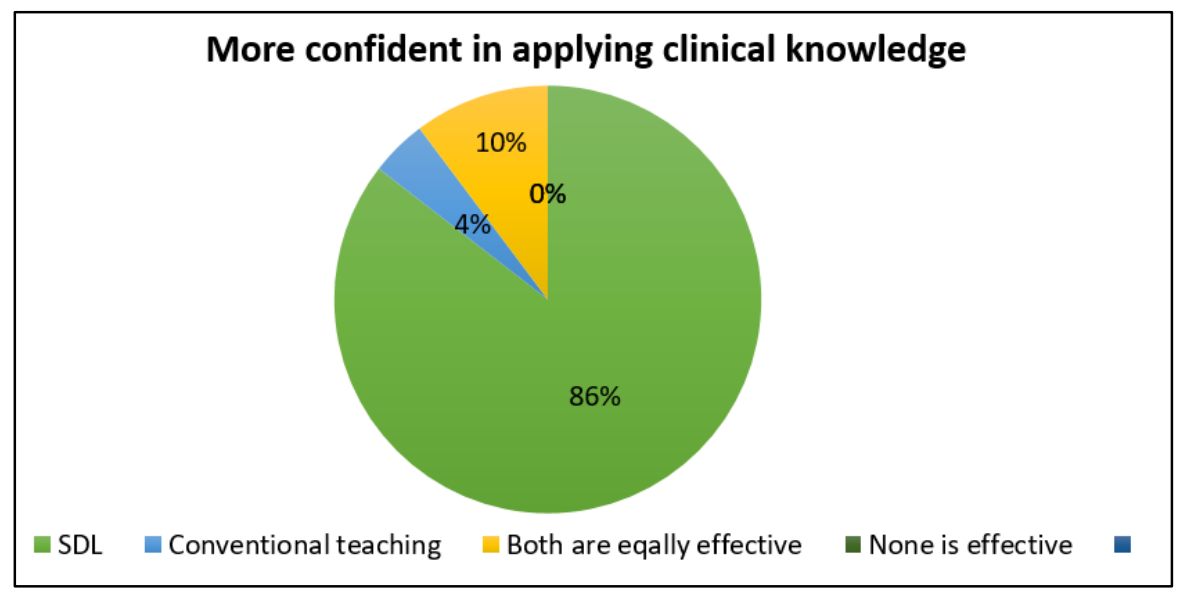

Fig. 5

Faculty perception: Perception analysis of faculty members is represented in fig. 6

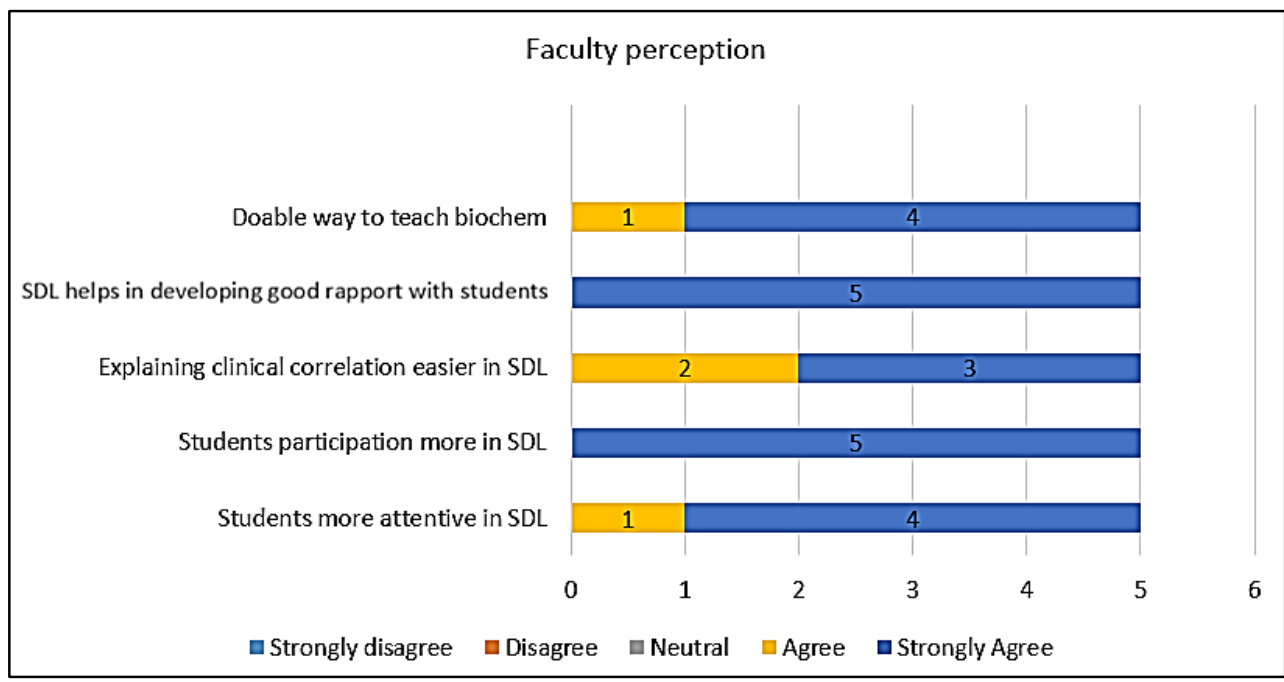

Fig. 6

\section{Discussion}

SDL can be an effective tool for teaching various topics in Biochemistry. Previous studies have mentioned that SDL is a good method for knowledge acquisition especially among first year undergraduate medical students. ${ }^{4}$ Research has also shown that SDL which incorporates case based learning is an effective method of introducing clinical correlation when conducted during the early years of undergraduate training in medicine. ${ }^{5}$

Ramsey et al $^{6}$ showed that knowledge of internist is inversely proportional to the gap between their board exam and current date of practice with the sharp decline of knowledge if this gap is more than 15 years. SDL is an important mode of learning which motivates learner to be a life long learner.

In the present study, an overwhelming majority of the students [84\%] have found SDL as a more interesting and enthusiastic way of learning a topic in biochemistry compared to $10 \%$ who feel conventional teaching in the form of didactic lecture is more interesting way to learn.

Majority of students found that SDL is an enjoyable form of learning and it gives ample opportunity to interact with the faculty. It is thus clear that anything which makes interaction with the faculty more intensive, makes the programme more enjoyable by the learner and develops more curiosity and interest in learners mind.

Despite finding SDL as a better way of learning the subject, some students still preferred conventional teaching of the topic in class room setting in the form of didactic lecture. This may be due to the fact that some students are Auditory learners and prefer listening to the faculty rather than actively participating in the teaching learning programme. Studies have already shown that acceptability of SDL is not universal and shows a major impact of culture of surrounding. ${ }^{7}$

One important feedback from many students was that SDL sessions gave them ample opportunity to recall and reinforce already learned topic. In their opinion students display better recall andthey get greater opportunity to learn 
from their peersafter attending a session where they have already read the topic.

"SDL is an excellent session to recall and strengthen already learned topic. This makes the concept clearer in our mind."

"During SDL sessions we have the opportunity to discuss the topic with peer group, this is creating more interest in the subject. I thoroughly enjoyed it."

Some of the students have admitted that discussion in small group in fish bowl methodology helped them to open up and speak in peer group which certainly boosted their self-esteem and confidence and helped them develop the communication skill.

$86 \%$ students found that application of clinical knowledge is easier when topic is learned in SDL module. Similar improvement in clinical skills have been reported in earlier studies. ${ }^{8,9}$

"I learned a lot of clinical things and it has motivated me enough to go and learn more about this topic"

They found it as a great opportunity to learn from discussion with the fellow colleague.

"It was the best session of my life. In this session everyone got the opportunity to talk. Those who had not read the topic had opportunity to listen and learn from fellow colleague discussion."

Some students said that SDL motivates them to do self study and boost their self confidence. At the same time there were good number of students who found that SDL is a labour intensive process and only competent facilitator can conduct it well.

Premkumar et al have reported that in order to promote SDL faculty development is important in implementing the changes. ${ }^{10}$

Faculty perception of SDL was positive.They found SDL more beneficial for the students and also found it easy to incorporate. All the faculties strongly agreed thatstudents actively participated in SDL and SDL session help to develop good rapport with the students.

All the faculty members agreed that explaining clinical correlation was easier under SDL (60\% strongly agreed and $40 \%$ agreed). $100 \%$ faculty agreed (with $80 \%$ faculty strongly agreeing) that SDL is a doable method for teaching Biochemistry. Faculty certainly are of opinion that prior preparation on their ends is essential.

\section{Conclusion}

SDL is certainly an effective mode of teaching certain topics in Biochemistry. Those topics which needs more of the faculty interaction can be chosen as a topic for SDL as this kind of programme gives ample opportunity to the students to learn the topic beforehand in guided fashion. Implementation of SDL should not be taken as challenge, wisely and timely planned SDL can turn out to be a boon for teaching certain topics in biochemistry in more effective and fascinating manner.

\section{Conflict of interest}

None.

\section{References}

1. Davis J. Education through self-directed learning. Aust Nurs Midwifery J 2015;23:26-7.

2. Hewitt-Taylor J. Teachers' and students' views on self-directed learning. Nurs Stand 2002;18-24;17(1):33-8.

3. Fry H, Jones A. Self directed learning in the undergraduate dental curriculum. Br Dent J. 1995;179(10):373-6.

4. Pai KM, Rao KR, Punja D, Kamath A. The effectiveness of self-directed learning (SDL) for teaching physiology to firstyear medical students. Australas Med J 2014;7(11):448-53.

5. Anderson SM, Helberg SB. Chart-based, case-based learning. S D Med. 2007;60(10).

6. Murad MH, Varkey P. Self-directed learning in health professions education. Ann Acad Med Singapore 2008;37(7):580-90.

7. Frambach JM, Driessen EW, Chan LC, Cees P M, van der Vleuten Rethinking the globalisation of problem based learning: how culture challenges self directed learning. Med Edu 2012;46:738-47

8. Considine J, Botti M, Thomas S. Effect of a self-directed learning package on emergency nurses' knowledge of assessment of oxygenation and use of supplemental oxygen. Nurs Health Sci 2005;7:199-208. doi: 10.1111/j.14422018.2005.00236.x.

9. Taylor EJ, Mamier I, Bahjri K, Anton T, Petersen F. Efficacy of a self-study programme to teach spiritual care. J Clin Nurs 2009;18:1131-40. doi:10.1111/j.1365-2702.2008.02526.x.

10. Premkumar K, Vinod E, Sathishkumar S. Self-directed learning readiness of Indian medical students: a mixed method study. BMC Med Educ 2018;18:134. doi:10.1186/s12909-0181244-9.

\section{Source of funding}

None. 